\title{
Aortic root surgery in the United States: A report from the Society of Thoracic Surgeons database
}

\author{
Sotiris C. Stamou, MD, PhD, ${ }^{\text {a }}$ Mathew L. Williams, MD, ${ }^{b}$ Tyler M. Gunn, MD, ${ }^{a}$ Robert C. Hagberg, MD, \\ Kevin W. Lobdell, MD, ${ }^{\mathrm{d}}$ and Nicholas T. Kouchoukos, MD
}

Objective: The purpose of the present study was to evaluate the early clinical outcomes of aortic root surgery in the United States.

\begin{abstract}
Methods: The Society of Thoracic Surgeons database was queried to identify all patients who had undergone aortic root replacement from 2004 to early $2010(\mathrm{n}=13,743)$. The median age was 58 years (range, 18-96); 3961 were women $(29 \%)$ and 12,059 were white $(88 \%)$. The different procedures included placement of a mechanical valve conduit $(n=4718,34 \%)$, stented pericardial $(n=879,6.4 \%)$ or porcine $(n=478,3.5 \%)$ bioprosthesis, stentless root $(\mathrm{n}=4309,31 \%)$, homograft $(\mathrm{n}=498,3.6 \%)$, and valve sparing root replacement $(\mathrm{n}=1918,14 \%)$.
\end{abstract}

Results: The median number of aortic root surgeries per site was 2 , and only $5 \%$ of sites performed $>16$ aortic root surgeries annually. An increased trend to use biostented (porcine or pericardial) valves during the study period (7\% in 2004 vs $14 \%$ in 2009). The operative (raw) mortality was greater among the patients with aortic stenosis $(6.2 \%)$ who had undergone aortic root replacement, independent of age. Mortality was greater in patients who had undergone concomitant valve or coronary artery bypass grafting or valve surgery $(21 \%)$. The lowest operative mortality was observed in patients who had undergone aortic valve sparing procedures $(1.9 \%)$.

Conclusions: Most cardiac centers performed aortic root surgery in small volumes. The unadjusted operative mortality was greater for patients $>80$ years old and those with aortic stenosis, regardless of age. Valve sparing root surgery was associated with the lowest mortality. A trend was seen toward an increased use of stented tissue valves from 2004 to 2009. (J Thorac Cardiovasc Surg 2015;149:116-22)

丹 Supplemental material is available online.

Aortic root replacement has historically associated with considerable morbidity and mortality. ${ }^{1,2}$ Recent technical advances in thoracic aortic surgery and improvements in cerebral and myocardial protection has improved outcomes $^{3}$; however, significant morbidity and mortality has remained. ${ }^{4}$ The contemporary outcomes of aortic root surgery in the United States are uncertain. The purpose of

From the Department of Cardiothoracic Surgery, ${ }^{\text {a }}$ University of Iowa Hospitals and Clinics, Iowa City, Iowa; Division of Thoracic and Cardiovascular Surgery, ${ }^{\text {b }}$ University of Louisville, Louisville, Ky; Hartford Hospital, ${ }^{\mathrm{c}}$ Hartford, Conn; Sanger Heart and Vascular Institute, ${ }^{\mathrm{d}}$ Carolinas Medical Center, Charlotte, NC; and Department of Cardiothoracic Surgery, ${ }^{e}$ Missouri Baptist Medical Center, St Louis, Mo.

Disclosures: Authors have nothing to disclose with regard to commercial support.

Read at The American Association for Thoracic Surgery Aortic Symposium, New York, New York, April 26-27, 2012.

Received for publication April 30, 2014; accepted for publication May 16, 2014; available ahead of print June 14, 2014.

Address for reprints: Sotiris C. Stamou, MD, PhD, Department of Cardiothoracic Surgery, 200 Hawkins Drive, SE 516 GH, Iowa City, IA 52242 (E-mail: sortiris-stamou@uiowa.edu).

$0022-5223 / \$ 36.00$

Copyright $($ c 2015 by The American Association for Thoracic Surgery

http://dx.doi.org/10.1016/j.jtcvs.2014.05.042 the present study was to better evaluate the early clinical outcomes and institutional characteristics of aortic root surgery in the United States.

\section{METHODS \\ Patients}

The patient population analyzed in the present study was obtained from the Society of Thoracic Surgeons (STS) Adult Cardiac Surgery Database (ACSD). ${ }^{5}$ The study included all patients aged $\geq 18$ years who had undergone aortic root replacement from January 1, 2004 to early $2010(\mathrm{n}=13,743)$. The data were collected using versions v2.52 (years 2004-2007) and v2.61 (years 2008-2010) of the STS database (available at: http://www.sts.org/quality-research-patient-safety/national-database/ database-managers/adult-cardiac-surgery-database/d).

The exclusion criteria were active or treated endocarditis, type A aortic dissections, and an aortic aneurysm with locations other than the ascending aorta only or ascending aorta and aortic arch. When comparing the valve types used for aortic root reconstruction, only mechanical, stented (porcine or pericardial), stentless, and homograft valves were included; patients with autograft, ring, or band annuloplasty or who had missing or mismatched valve types were excluded. Patients with an unreported age were also excluded when comparing the operative outcomes by age. Previous reoperation was defined as any previous open heart procedure, such as previous coronary artery bypass grafting (CABG) and valve or other cardiac, great vessel, or congenital surgery. Univariate analysis was used to compare the surgical outcomes in the presence of preoperative conditions and concomitant procedures. When comparing the outcomes in the presence of aortic stenosis, increased age, concomitant $\mathrm{CABG}$, concomitant valve, concomitant $\mathrm{CABG}$ and valve procedure, and 


\section{Abbreviations and Acronyms \\ ACSD $=$ Adult Cardiac Surgery Database \\ $\mathrm{CABG}=$ coronary artery bypass grafting \\ LOS = length of stay \\ STS $=$ Society of Thoracic Surgeons}

concomitant aortic aneurysm repair, any missing data in the area of interest were imputed as negative.

\section{RESULTS}

\section{Patient and Operative Characteristics}

The present study included 13,746 patients who had undergone aortic root surgery from 2004 to 2010 (Table 1). The patient ages in the study population ranged from 18 to 96 years (median, 58 years); 3961 were women $(29 \%)$ and 12,059 were white $(87 \%)$. A total of 1918 patients had undergone a valve sparing root procedure $(15 \%)$ and 11,825 patients had undergone root reconstruction with a valve conduit. The prevalence of the various procedures was as follows: mechanical valve conduit, $37 \%$; stented pericardial, $7 \%$ or porcine bioprosthesis, $4 \%$; stentless root, $33 \%$; and homograft, $4 \%$. A total of 2724 patients had previously undergone cardiac surgery $(21 \%)$. Concomitant procedures included CABG $(\mathrm{n}=3811,30 \%)$ and mitral and/or tricuspid valve surgery $(\mathrm{n}=1201,9 \%)$.

\section{Morbidity and Mortality}

The overall operative raw mortality of all patients undergoing aortic root surgery was $4.2 \%$. The outcomes of patients with aortic stenosis, increased age, previous medial sternotomy, the use of select concomitant procedures, and various aortic root procedures are listed in Table 2. Operative mortality increased with patient age from $2.4 \%$ in patients $<60$ years old to $15 \%$ in patients $>80$ years old (Table 2). Aortic valve sparing root repair had the lowest incidence of operative mortality and in-hospital stroke. Concomitant procedures were associated with greater operative mortality, a longer length of stay (LOS), and increases in surgical complications, such as stroke, reoperation for bleeding, acute renal failure, and acute renal failure requiring dialysis (see Appendix Tables E2-E4). Operative mortality was greater among patients with aortic stenosis $(6.2 \%)$ than in those without $(3.5 \%)$ who had undergone aortic root replacement (Table 3). The incidence of acute renal failure, renal failure requiring dialysis, and LOS were increased in patients with aortic stenosis.

Operative mortality, LOS $>14$ days, and total intensive care unit stay worsened significantly with increased patient age (Table 4 ). The operative mortality of patients $\geq 80$ years was $15 \%$ and the rate of $\operatorname{LOS}>14$ days was $23 \%$ compared with $2.3 \%$ and $6.1 \%$, respectively, in patients $<60$ years. The proportion of female patients increased from $23 \%$ to $51 \%$ in patients $<60$ years old and those $>80$ years, respectively. In-hospital reoperations for any reason were more frequent in patients of increasing age.

\section{Redo Sternotomy and Concomitant Procedures}

Operative mortality and LOS $>14$ days was greater in patients with previous sternotomy $(8.9 \%$ and $15 \%)$ compared with those without $(3.5 \%$ and $8.6 \%$; Appendix Table E1). Concomitant procedures increased the operative mortality, rates of reoperation for bleeding, acute renal failure, and LOS > 14 days (Appendix Tables E2 and E3). The operative mortality of concomitant $\mathrm{CABG}$, valve (mitral or tricuspid), and CABG and valve was $9.5 \%$, $13 \%$, and $21 \%$, respectively (Table 3 ). The rate of acute renal failure and LOS $>14$ days was $9.5 \%$ and $16 \%$ with concomitant $\mathrm{CABG}, 13 \%$ and $22 \%$ with concomitant valve surgery, and $17 \%$ and $30 \%$ for concomitant $\mathrm{CABG}$ and valve surgery, respectively.

\section{Prosthesis Preference}

Aortic valve sparing aortic root procedures resulted in an operative mortality of $1.9 \%$ (Table 1 ), which was less than that for mechanical valve conduit $(3.8 \%)$, stented tissue valve conduit $(5.6 \%)$, stentless root $(5.8 \%)$, and homograft $(7.6 \%)$ procedures (Table 5). The incidence of stroke was greatest in the homograft $(4.0 \%)$ than in the mechanical valve conduit $(2.0 \%)$, stented tissue valve $(3.1 \%)$, stentless root $(2.2 \%)$, and aortic valve sparing $(2.3 \%)$ procedures. The rates of acute renal failure, reoperation for bleeding, hemodialysis, and LOS $>14$ days were similar among the aortic root procedures. Mechanical (44\%) and biostentless $(40 \%)$ valves were the most frequently used, and biostented pericardial $(8.1 \%)$, biostented porcine $(4.4 \%)$, and homograft $(4.4 \%)$ valves were less common.

\section{Trends in Aortic Root Surgery}

The percentage of patients undergoing aortic root surgery with mechanical, biostented, stentless, and homograft valves or valve sparing procedures were compared between 2004 and 2009 (Appendix Table E5). The mechanical and biostented valves were the most frequently used prosthesis from 2004 to 2009, with stentless and homograft valves less common after 2006. The usage of biostented valves has nearly doubled in 5 years. The proportion of valve sparing aortic root procedures has remained approximately $15 \%$.

\section{DISCUSSION}

The present study included a large contemporary cohort evaluating aortic root replacement surgery trends in the United States reported to date with 13,746 patients included from 2004 to 2010 . A recently published study by Williams and colleagues ${ }^{4}$ analyzed patients reported in the STS 
TABLE 1. Patient population and outcomes stratified by aortic root procedure

\begin{tabular}{|c|c|c|c|}
\hline \multirow[b]{2}{*}{ Characteristic } & \multirow[b]{2}{*}{ Overall $(n=13,743)$} & \multicolumn{2}{|c|}{ Root reconstruction } \\
\hline & & Valve sparing $(n=1918)$ & Valve conduit $(n=11,825)$ \\
\hline Age (y) & $58(48-69)$ & $51(39-63)$ & $59(49-69)$ \\
\hline Female gender & 28.82 & 27.48 & 29.04 \\
\hline \multicolumn{4}{|l|}{ Race } \\
\hline White & 87.75 & 86.08 & 88.02 \\
\hline Black & 6.05 & 7.56 & 5.80 \\
\hline Asian & 1.48 & 1.82 & 1.42 \\
\hline Hispanic & 1.30 & 1.09 & 1.34 \\
\hline \multicolumn{4}{|l|}{ Demographic region } \\
\hline Midwest & 35.26 & 37.75 & 34.86 \\
\hline Northeast & 16.88 & 15.85 & 17.05 \\
\hline South & 28.80 & 24.50 & 29.50 \\
\hline West & 19.06 & 21.90 & 18.60 \\
\hline BMI $\left(\mathrm{kg} / \mathrm{m}^{2}\right)$ & $28(25-32)$ & $27(24-31)$ & $28(25-32)$ \\
\hline Hypertension & 66.34 & 59.65 & 67.53 \\
\hline Current or recent smoker & 17.54 & 17.36 & 17.57 \\
\hline Hypercholesterolemia & 48.89 & 41.24 & 50.13 \\
\hline Chronic lung disease & 16.55 & 11.31 & 17.40 \\
\hline \multicolumn{4}{|l|}{ Renal function status } \\
\hline $\mathrm{GFR} \geq 90 \mathrm{~mL} / \mathrm{min} / 1.73 \mathrm{~m}^{2}$ & 28.81 & 37.7 & 27.37 \\
\hline GFR $60-89 \mathrm{~mL} / \mathrm{min} / 1.73 \mathrm{~m}^{2}$ & 52.25 & 51.25 & 52.41 \\
\hline GFR $30-59 \mathrm{~mL} / \mathrm{min} / 1.73 \mathrm{~m}^{2}$ & 17.04 & 10.22 & 18.15 \\
\hline $\mathrm{GFR} \leq 29 \mathrm{~mL} / \mathrm{min} / 1.73 \mathrm{~m}^{2}$ & 0.97 & 0.31 & 1.07 \\
\hline Dialysis & 0.92 & 0.52 & 0.99 \\
\hline Immunosuppressive treatment & 2.13 & 1.67 & 2.21 \\
\hline Diabetes mellitus & 13.03 & 8.17 & 13.83 \\
\hline Peripheral vascular disease & 14.17 & 14.13 & 14.18 \\
\hline Cerebrovascular disease & 9.18 & 7.40 & 9.47 \\
\hline History of myocardial infarction & 8.61 & 5.26 & 9.16 \\
\hline History of congestive heart failure & 25.85 & 12.51 & 28.02 \\
\hline Any previous cardiovascular surgery & 19.82 & 13.45 & 20.85 \\
\hline Previous CABG & 3.46 & 2.03 & 3.69 \\
\hline Previous valve procedure & 12.46 & 6.62 & 13.40 \\
\hline Preoperative cardiogenic shock & 0.27 & 0.21 & 0.28 \\
\hline \multicolumn{4}{|l|}{ Procedure status } \\
\hline Elective & 83.08 & 89.42 & 82.05 \\
\hline Urgent & 16.92 & 10.58 & 17.95 \\
\hline \multicolumn{4}{|l|}{ Urgent reason } \\
\hline Anatomy & 29.11 & 46.80 & 27.41 \\
\hline Valve dysfunction & 37.10 & 20.20 & 38.72 \\
\hline Congestive heart failure & 22.66 & 15.76 & 23.32 \\
\hline Concomitant CABG & 25.65 & 14.91 & 27.39 \\
\hline Concomitant mitral valve procedure & 6.72 & 6.36 & 6.78 \\
\hline Concomitant arrhythmia correction & 5.59 & 5.27 & 5.64 \\
\hline Operation time (h) & $5.3(4.3-6.8)$ & $5.7(4.5-7.1)$ & $5.3(4.2-6.7)$ \\
\hline Crossclamp time (min) & $139(110-178)$ & $157(107-208)$ & $138(110-174)$ \\
\hline
\end{tabular}

Data presented as median (25th to 75th percentiles) or \%. BMI, Body mass index; GFR, glomerular filtration rate; CABG, coronary artery bypass grafting.

ACSD from 2004 to 2009 who had undergone proximal aortic replacement, including combinations of aortic root ascending aorta with or without involvement of the aortic arch. Our study focused specifically on aortic root replacement and omitted repair of the ascending aorta and aortic arch. Multiple important findings were evident from our study.

\section{Principal Findings}

Aortic root repair remains an uncommon procedure for most surgical centers, with only a few sites reporting large annual case volumes. In the present study, the median number of aortic root surgeries per site was 2, and only $5 \%$ of institutions participating in the STS ACSD performed $>16$ aortic root surgeries in 2009 (Figure 1). 
TABLE 2. Outcomes stratified by postoperative outcomes

\begin{tabular}{|c|c|c|c|c|c|c|}
\hline Variable & Operative mortality & Stroke & Reoperation for bleeding & Acute renal failure & Hemodialysis & $\operatorname{LOS}>14 \mathrm{~d}$ \\
\hline Aortic stenosis & 6.24 & 2.24 & 5.29 & 7.32 & 3.29 & 11.65 \\
\hline \multicolumn{7}{|l|}{ Age (y) } \\
\hline$<60$ & 2.35 & 1.34 & 5.03 & 3.44 & 1.36 & 6.11 \\
\hline $60-69$ & 4.58 & 2.35 & 6.31 & 6.31 & 2.29 & 9.88 \\
\hline $70-79$ & 8.02 & 3.62 & 7.11 & 8.55 & 4.36 & 16.82 \\
\hline$\geq 80$ & 15.41 & 3.78 & 6.83 & 11.48 & 5.09 & 23.11 \\
\hline \multicolumn{7}{|l|}{ Concomitant procedures } \\
\hline CABG & 9.45 & 3.46 & 7.94 & 9.53 & 4.37 & 15.94 \\
\hline Valve (mitral or tricuspid) & 13.29 & 2.77 & 8.80 & 13.00 & 5.26 & 22.47 \\
\hline CABG and valve & 21.40 & 4.01 & 9.70 & 16.72 & 7.02 & 30.43 \\
\hline Aortic aneurysm & 2.99 & 2.04 & 5.81 & 4.27 & 1.72 & 8.39 \\
\hline Redo sternotomy & 8.92 & 2.64 & 7.20 & 8.52 & 4.00 & 14.68 \\
\hline \multicolumn{7}{|l|}{ Aortic root procedure } \\
\hline Mechanical valve conduit & 3.76 & 2.01 & 6.17 & 4.80 & 1.86 & 8.62 \\
\hline Stented tissue valve conduit & 5.69 & 3.07 & 6.50 & 6.80 & 2.16 & 12.86 \\
\hline Stentless root & 5.82 & 2.17 & 5.54 & 6.83 & 3.28 & 12.00 \\
\hline Homograft & 7.63 & 4.03 & 6.36 & 6.14 & 3.18 & 10.17 \\
\hline Aortic valve sparing & 1.88 & 2.26 & 6.00 & 5.80 & 2.40 & 10.45 \\
\hline
\end{tabular}

Data presented as \%. LOS, Length of stay; $C A B G$, coronary artery bypass grafting.

Another finding of our study was an increasing trend during aortic root reconstruction to use biostented (porcine or pericardial) rather than mechanical, homograft, or biostentless valves (Figure 2). In 2009, 14\% of patients received a biostented valve compared with only $7.6 \%$ received a biostented valve in 2004. The decision of whether to implant a bioprosthesis or a mechanical valve has largely been dependent on the age of the patient and their preference toward 1 of 2 sets of complications: the requirement for chronic anticoagulation therapy and its complications in mechanical valves or structural valve deterioration with a bioprosthesis. ${ }^{6}$

TABLE 3. Outcomes stratified by aortic stenosis

\begin{tabular}{|c|c|c|c|}
\hline \multirow[b]{2}{*}{ Characteristic } & \multirow[b]{2}{*}{ Overall $(n=13,743)$} & \multicolumn{2}{|c|}{ Aortic stenosis } \\
\hline & & No $(\mathbf{n}=\mathbf{8 5 6 8})$ & Yes $(n=5175)$ \\
\hline Age (y) & $58(48-69)$ & $56(45-67)$ & $62(52-72)$ \\
\hline Female gender & 28.82 & 23.90 & 36.97 \\
\hline BMI $\left(\mathrm{kg} / \mathrm{m}^{2}\right)$ & $28(25-32)$ & $28(24-31)$ & $28(25-33)$ \\
\hline Hypertension & 66.43 & 66.29 & 66.65 \\
\hline Hypercholesterolemia & 48.89 & 44.09 & 56.83 \\
\hline Chronic lung disease & 16.55 & 15.51 & 18.28 \\
\hline Diabetes mellitus & 13.03 & 9.18 & 19.40 \\
\hline Any previous cardiovascular surgery & 19.82 & 19.40 & 20.52 \\
\hline \multicolumn{4}{|l|}{ Procedure status } \\
\hline Elective & 83.08 & 83.91 & 81.7 \\
\hline Urgent & 16.92 & 16.09 & 18.3 \\
\hline \multicolumn{4}{|l|}{ Urgent reason } \\
\hline Anatomy & 29.11 & 36.48 & 18.37 \\
\hline Valve dysfunction & 37.10 & 32.56 & 43.72 \\
\hline Congestive heart failure & 22.66 & 20.09 & 26.40 \\
\hline Operation time (h) & $5.3(4.3-6.7)$ & $5.4(4.3-6.8)$ & $5.2(4.2-6.7)$ \\
\hline Crossclamp time (min) & $139(110-178)$ & $139(109-179)$ & $139(111-176)$ \\
\hline ICU stay $(\mathrm{h})$ & $45.8(24.0-88.2)$ & $45.0(24.0-75.0)$ & $47.5(24.9-96.0)$ \\
\hline LOS $>14 \mathrm{~d}$ & 9.78 & 8.65 & 11.64 \\
\hline Septicemia in hospital & 1.70 & 1.30 & 2.36 \\
\hline Cardiac arrest in hospital & 3.38 & 2.80 & 4.33 \\
\hline Reoperation, any reason & 10.88 & 10.62 & 11.30 \\
\hline Operative mortality & 4.56 & 3.54 & 6.24 \\
\hline
\end{tabular}

Data presented as median (25th to 75 th percentiles) or \%. BMI, Body mass index; ICU, intensive care unit; $L O S$, length of stay. 
TABLE 4. Outcomes stratified by age group

\begin{tabular}{|c|c|c|c|c|c|}
\hline \multirow[b]{2}{*}{ Characteristic } & \multirow[b]{2}{*}{ Overall $(n=13,743)$} & \multicolumn{4}{|c|}{ Age (y) } \\
\hline & & $<60(n=7262)$ & $\geq 60$ but $<70(n=3361)$ & $\geq 70$ but $<80(n=2432)$ & $\geq 80(n=688)$ \\
\hline Age (y) & $58(48-69)$ & $49(40-54)$ & $64(62-67)$ & $74(72-76)$ & $82(81-84)$ \\
\hline Female gender & 28.82 & 22.60 & 28.86 & 41.00 & 51.53 \\
\hline $\mathrm{BMI}\left(\mathrm{kg} / \mathrm{m}^{2}\right)$ & $28(25-32)$ & $28(25-32)$ & $28(25-32)$ & $27(24-31)$ & $26(23-29)$ \\
\hline Hypertension & 66.43 & 54.89 & 76.67 & 81.66 & 84.30 \\
\hline Hypercholesterolemia & 48.89 & 37.69 & 59.51 & 63.90 & 62.21 \\
\hline Chronic lung disease & 16.55 & 11.88 & 20.59 & 23.23 & 22.53 \\
\hline Diabetes mellitus & 13.03 & 8.46 & 17.23 & 19.57 & 17.73 \\
\hline Any previous cardiovascular surgery & 19.82 & 20.52 & 18.12 & 19.28 & 22.67 \\
\hline \multicolumn{6}{|l|}{ Procedure status } \\
\hline Elective & 83.08 & 85.27 & 81.82 & 80.47 & 75.29 \\
\hline Urgent & 16.92 & 14.73 & 18.18 & 19.53 & 24.71 \\
\hline \multicolumn{6}{|l|}{ Urgent reason } \\
\hline Anatomy & 29.11 & 29.81 & 29.30 & 30.32 & 20.59 \\
\hline Valve dysfunction & 37.10 & 39.07 & 35.68 & 35.79 & 33.53 \\
\hline Congestive heart failure & 22.66 & 21.21 & 22.42 & 21.89 & 34.71 \\
\hline Operation time $(\mathrm{h})$ & $5.3(4.3-6.7)$ & $5.2(4.2-6.6)$ & $5.4(4.3-6.8)$ & $5.6(4.4-7.0)$ & $5.5(4.3-7.0)$ \\
\hline Crossclamp time (min) & $139(110-178)$ & $138(109-177)$ & $140(111-178)$ & $143(111-180)$ & $138(109-178)$ \\
\hline ICU stay (h) & $45.8(24.0-88.2)$ & $41.0(23.0-69.0)$ & $48.0(25.2-93.0)$ & $56.7(29.0-120.0)$ & $76.0(44.0-167.0)$ \\
\hline $\mathrm{LOS}>14 \mathrm{~d}$ & 9.78 & 6.11 & 9.88 & 16.82 & 23.11 \\
\hline Reoperation, any reason & 10.88 & 8.88 & 11.72 & 13.94 & 17.01 \\
\hline Operative mortality & 4.56 & 2.35 & 4.58 & 8.02 & 15.41 \\
\hline
\end{tabular}

Data presented as median (25th to 75 th percentiles) or \%. BMI, Body mass index; $I C U$, intensive care unit; $L O S$, length of stay.

Two large randomized clinical trials, the Edinburgh Heart Valve Trial $^{7}$ and the Department of Veterans Affairs trial, ${ }^{8}$ found no statistically significant differences between mechanical and bioprosthesis valves for systemic embolism, valve thrombosis, and prosthetic endocarditis or survival. However, increased rates of bleeding were found in patients with mechanical valves, and bioprosthetic aortic valve replacement with structural valve deterioration was greater

TABLE 5. Outcomes stratified by valve type

\begin{tabular}{|c|c|c|c|c|c|c|}
\hline \multirow[b]{2}{*}{ Characteristic } & \multirow[b]{2}{*}{$\begin{array}{c}\text { Overall } \\
(\mathbf{n}=\mathbf{1 0 , 7 3 7})\end{array}$} & \multirow[b]{2}{*}{$\begin{array}{c}\text { Mechanical } \\
(n=4683)\end{array}$} & \multicolumn{4}{|c|}{ Biostented } \\
\hline & & & $\begin{array}{l}\text { Porcine } \\
(n=468)\end{array}$ & $\begin{array}{c}\text { Pericardial } \\
(n=867)\end{array}$ & $\begin{array}{c}\text { Biostentless } \\
(\mathrm{n}=\mathbf{4 2 4 3})\end{array}$ & $\begin{array}{c}\text { Homograft } \\
(n=472)\end{array}$ \\
\hline Age (y) & $60(49-60)$ & $53(44-60)$ & $67(58-76)$ & $66(57-74)$ & $66(57-74)$ & $60(48-70)$ \\
\hline Female gender & 29.48 & 21.27 & 35.47 & 29.41 & 37.59 & 32.20 \\
\hline BMI $\left(\mathrm{kg} / \mathrm{m}^{2}\right)$ & $28(25-32)$ & $28(25-32)$ & $27(24-30)$ & $28(25-31)$ & $28(25-32)$ & $27(24-31)$ \\
\hline Hypertension & 68.08 & 63.32 & 72.44 & 72.32 & 72.28 & 65.47 \\
\hline Hypercholesterolemia & 50.56 & 43.84 & 54.06 & 56.86 & 56.63 & 47.67 \\
\hline Chronic lung disease & 17.59 & 15.43 & 19.66 & 17.76 & 20.08 & 14.41 \\
\hline Diabetes mellitus & 13.90 & 9.83 & 18.80 & 16.96 & 17.66 & 11.02 \\
\hline Any previous cardiovascular surgery & 20.80 & 21.76 & 20.09 & 16.26 & 19.49 & 31.99 \\
\hline \multicolumn{7}{|l|}{ Procedure status } \\
\hline Elective & 81.81 & 83.06 & 81.62 & 79.93 & 81.43 & 76.48 \\
\hline Urgent & 18.19 & 16.94 & 18.38 & 20.07 & 18.57 & 23.52 \\
\hline \multicolumn{7}{|l|}{ Urgent reason } \\
\hline Anatomy & 26.83 & 32.87 & 20.93 & 26.44 & 23.35 & 13.51 \\
\hline Valve dysfunction & 39.27 & 38.16 & 44.19 & 43.68 & 35.41 & 63.96 \\
\hline Congestive heart failure & 23.45 & 18.89 & 25.58 & 20.69 & 29.44 & 16.22 \\
\hline Operation time (h) & $5.3(4.2-6.7)$ & $5.2(4.2-6.6)$ & $5.2(4.1-6.6)$ & $5.3(4.2-6.8)$ & $5.4(4.3-6.7)$ & $5.3(4.3-7.0)$ \\
\hline Crossclamp time (min) & $137(110-174)$ & $132(106-168)$ & $127(97-169)$ & $137(104-173)$ & $142(115-178)$ & $148(115-188)$ \\
\hline ICU stay $(\mathrm{h})$ & $46.5(24.4-92.2)$ & $46.0(24.0-76.23)$ & $57.9(36.0-116.3)$ & $48.5(25.5-95.0)$ & $47.0(24.25-96.0)$ & $44.4(24.2-93.0)$ \\
\hline $\operatorname{LOS}>14 \mathrm{~d}$ & 10.55 & 8.62 & 16.03 & 11.19 & 12.00 & 10.17 \\
\hline Reoperation, any reason & 11.47 & 11.27 & 13.68 & 11.42 & 11.38 & 12.29 \\
\hline Operative mortality & 4.98 & 3.76 & 7.48 & 4.73 & 5.82 & 7.63 \\
\hline
\end{tabular}

Data presented as median (25th to 75 th percentiles) or \%. BMI, Body mass index; ICU, intensive care unit; $L O S$, length of stay. 


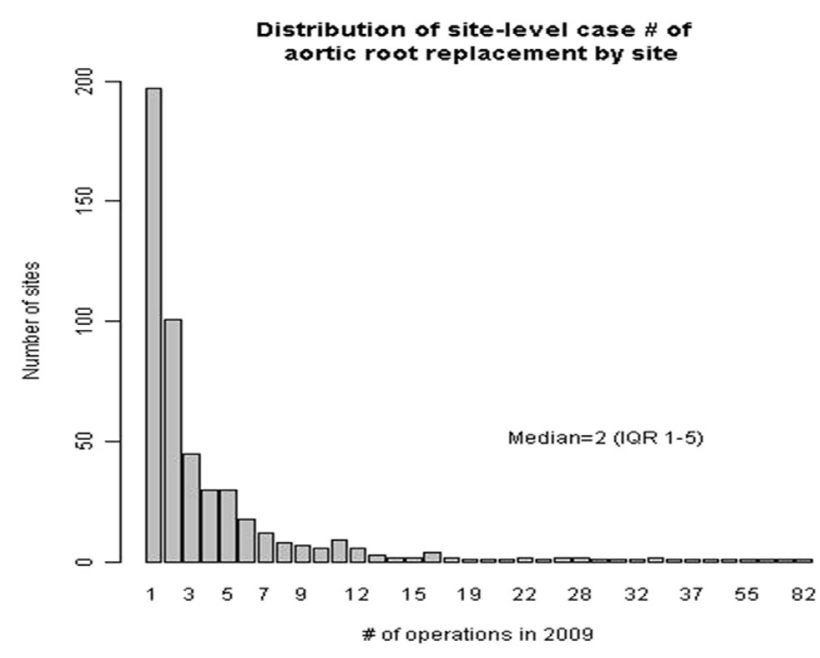

FIGURE 1. Distribution of aortic root replacements by site (year 2009). $I Q R$, Interquartile range.

in patients aged $<65$ years in the Veterans Affairs trial. Stentless bioprosthetic aortic valve replacement has been theorized to provide benefits in valve hemodynamics and clinical outcomes; however, these potential advantages compared with stented bioprosthetic valves could be offset by the increased technical complexity of implantation, with longer crossclamp and cardiopulmonary bypass times. The presence of calcification, which will increase the complexity and morbidity of redo aortic root replacement, could also be an issue. A recent meta-analysis of randomized controlled trials revealed that stentless valves do not increase perioperative risk; however, they did not exhibit benefits in hemodynamics or clinical outcomes compared with stented valves.'

\section{Operative Mortality}

Mortality was greater in patients $>80$ years old $(15 \%)$ and those who had undergone concomitant valve or CABG and valve surgery $(21 \%)$. These findings are consistent with the proximal aortic replacement predictors of operative mortality

\section{AORTIC ROOT SURGERY TRENDS}

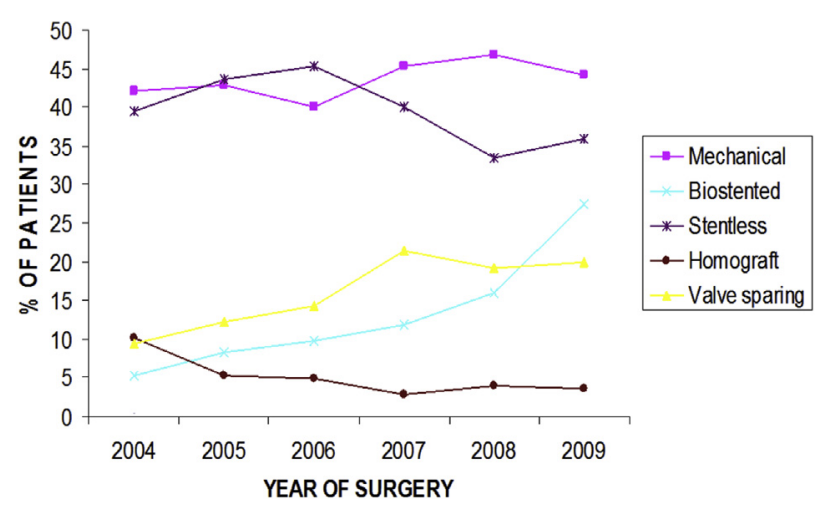

FIGURE 2. Trends in aortic root replacement prosthesis choice by year of surgery. reported by Williams and colleagues, ${ }^{4}$ who reported risk-adjusted odds ratios of 2.14 for concomitant CABG among all patients and 1.44 for patients aged $>70$ years undergoing elective procedures. In our study, all concomitant procedures increased operative mortality, including additive mitral or tricuspid valve repair, additive mitral or tricuspid valve repair with $\mathrm{CABG}$, and $\mathrm{CABG}$ alone. Increased technical complexity, coupled with the substantially longer cardiopulmonary bypass and aortic crossclamp times, likely contributed to worse short-term outcomes.

The overall unadjusted operative mortality was greater in patients with aortic stenosis $(6.2 \%)$ than in those without $(3.5 \%)$ who had undergone aortic root replacement, regardless of age. Published data are sparse regarding the operative mortality of aortic root surgery in the presence of aortic stenosis. The pathophysiologic consequences of prolonged aortic stenosis, including myocardial hypertrophy and decreased left ventricular cardiac output, likely contribute to the greater operative mortality.

The lowest operative morality was observed in patients who had undergone aortic valve sparing procedures $(1.88 \%)$ compared with root reconstruction with a valve conduit $(5.0 \%)$. Two types of aortic valve sparing root replacement have been common: aortic root remodeling, known as the Yacoub procedure, ${ }^{10}$ and the more commonly performed reimplantation of the aortic valve, known as the David procedure. ${ }^{11}$ The low operative mortality reported in the present study is consistent with other recently published single-center studies. Kvitting and colleagues ${ }^{12}$ reported an operative mortality of $0.9 \%$ in 233 patients undergoing David valve-sparing aortic root replacement at Stanford University from 1993 to 2009. Another study of 236 patients who had undergone a David procedure from 1997 to 2011 reported an operative mortality of $1.7 \% .^{13}$ The mortality of David procedures has been greater in highrisk situations. In a study of 110 patients with acute type A dissection, severe aortic insufficiency, or reoperation, the operative mortality was reported as $5.4 \%$ and $8.2 \%$ for the David and Bentall procedure patients, respectively. ${ }^{14}$

\section{Study Limitations}

Although the STS ACSD encompasses the significant majority of cardiac surgical centers in the United States and contains the most recent reported results, those cases performed at nonparticipating centers were not included in our analysis. The data regarding underlying aortic pathologic factors and variations in surgical technique, including connective tissue diagnosis, aortic diameter, and adjunct monitoring of cerebral perfusion, were not available for incorporation into the present study.

\section{Clinical Implications}

Most surgical centers in the United States performed $\leq 2$ aortic root procedures in 2009, and only $5 \%$ of sites 
performed $>16$ operations, making it more difficult to maintain the expertise required to maximize successful outcomes for these technically complex procedures. Studies have shown that an inverse association exists between hospital procedure volume and postoperative mortality for surgeries of the aortic root, ${ }^{6,7}$ and this is likely true for aortic root replacement, one of the most technically complex, yet least commonly performed, cardiac operations.

The lowest operative mortality was observed for patients who had undergone aortic valve sparing procedures, suggesting that the David procedure should continue to be the favored alternative, if technically feasible. Greater operative mortality was found among patients with aortic stenosis, patients $>80$ years old, and those who had undergone concomitant valve or CABG and valve surgery. Caution should be applied when considering aortic root replacement for patients with aortic stenosis without an aortic root aneurysm, because a less complex operation might be a safer alternative. Additional characterization of the STS ACSD aortic root surgery outcomes in these areas could assist in modifying the screening thresholds for elective repair, which should result in improved outcomes.

\section{References}

1. Zehr KJ, Orszulak TA, Mullany CJ, Matloobi A, Daly RC, Dearani JA, et al. Surgery for aneurysms of the aortic root: a 30-year experience. Circulation. 2004;110:1364-71.

2. Centofanti P, Flocco R, Ceresa F, Attisani M, La Torre M, Weltert L, et al. Is surgery always mandatory for type A aortic dissection? Ann Thorac Surg. 2006;82:1658-63; discussion 1664.
3. Krahenbuhl ES, Immer FF, Stalder M, Englberger L, Eckstein FS, Schmidli J, et al. Technical advances improved outcome in patients undergoing surgery of the ascending aorta and/or aortic arch: ten years' experience. Eur J Cardiothorac Surg. 2008;34:595-9.

4. Williams JB, Peterson ED, Zhao Y, O'Brien SM, Andersen ND, Miller DC, et al. Contemporary results for proximal aortic replacement in North America. J Am Coll Cardiol. 2012;60:1156-62.

5. Ferguson TB Jr, Dziuban SW Jr, Edwards FH, Eiken MC, Shroyer AL, Pairolero PC, et al. The STS national database: current changes and challenges for the new millennium. Committee to Establish a National Database in Cardiothoracic Surgery, the Society of Thoracic Surgeons. Ann Thorac Surg. 2000;69:680-91.

6. Rahimtoola SH. Choice of prosthetic heart valve in adults: an update. J Am Coll Cardiol. 2010;55:2413-26.

7. Oxenham H, Bloomfield P, Wheatley DJ, Lee RJ, Cunningham J, Prescott RJ, et al. Twenty year comparison of a Bjork-Shiley mechanical heart valve with porcine bioprostheses. Heart. 2003;89:715-21.

8. Hammermeister K, Sethi GK, Henderson WG, Grover FL, Oprian C, Rahimtoola $\mathrm{SH}$, et al. Outcomes 15 years after valve replacement with a mechanical versus a bioprosthetic valve: final report of the Veterans Affairs randomized trial. J Am Coll Cardiol. 2000;36:1152-8.

9. Murtuza B, Pepper JR, Jones C, Nihoyannopoulos P, Darzi A, Athanasiou T, et al. Does stentless aortic valve implantation increase perioperative risk? A critical appraisal of the literature and risk of bias analysis. Eur J Cardiothorac Surg. 2011;39:643-52.

10. Sarsam MA, Yacoub M. Remodeling of the aortic valve annulus. J Thorac Cardiovasc Surg. 1993;105:435-8.

11. David TE, Feindel CM. An aortic valve-sparing operation for patients with aortic incompetence and aneurysm of the ascending aorta. J Thorac Cardiovasc Surg. 1992;103:617-21; discussion 622.

12. Kvitting JP, Kari FA, Fischbein MP, Liang DH, Beraud AS, Stephens EH, et al. David valve-sparing aortic root replacement: equivalent mid-term outcome for different valve types with or without connective tissue disorder. $J$ Thorac Cardiovasc Surg. 2013;145:117-26, 127.e1-5; discussion 126-7.

13. Liebrich M, Kruszynski MK, Roser D, Meisner C, Doll KN, Hemmer WB, et al. The David procedure in different valve pathologies: a single-center experience in 236 patients. Ann Thorac Surg. 2013;95:71-6.

14. Kerendi F, Guyton RA, Vega JD, Kilgo PD, Chen EP. Early results of valve-sparing aortic root replacement in high-risk clinical scenarios. Ann Thorac Surg. 2010;89:471-6; discussion 477-8. 
APPENDIX TABLE E1. Outcomes by redo sternotomy

\begin{tabular}{|c|c|c|c|}
\hline \multirow[b]{2}{*}{ Characteristic } & \multirow[b]{2}{*}{ Overall $(n=13,743)$} & \multicolumn{2}{|c|}{ Redo sternotomy } \\
\hline & & No $(n=11,019)$ & Yes $(n=2724)$ \\
\hline Age (y) & $58(48-69)$ & $59(48-69)$ & $57(45-68)$ \\
\hline Female gender & 28.82 & 28.97 & 28.23 \\
\hline BMI $\left(\mathrm{kg} / \mathrm{m}^{2}\right)$ & $28(25-32)$ & $28(25-32)$ & $28(24-32)$ \\
\hline Cerebrovascular disease & 9.18 & 7.57 & 15.71 \\
\hline Diabetes mellitus & 13.03 & 12.49 & 15.24 \\
\hline Congestive heart failure & 25.85 & 23.42 & 35.68 \\
\hline \multicolumn{4}{|l|}{ Procedure status } \\
\hline Elective & 83.08 & 84.20 & 78.52 \\
\hline Urgent & 16.92 & 15.80 & 21.48 \\
\hline \multicolumn{4}{|l|}{ Urgent reason } \\
\hline Anatomy & 29.11 & 32.40 & 19.32 \\
\hline Valve dysfunction & 37.10 & 32.57 & 50.60 \\
\hline Congestive heart failure & 22.66 & 22.11 & 24.27 \\
\hline Operation time $(\mathrm{h})$ & $5.3(4.3-6.7)$ & $5.1(4.2-6.4)$ & $6.5(5.2-8.3)$ \\
\hline Crossclamp time (min) & $139(110-178)$ & $136(108-174)$ & $152(118-194)$ \\
\hline ICU stay (h) & $45.8(24.0-88.2)$ & $45.0(24.0-75.0)$ & $50.0(25.5-118.0)$ \\
\hline LOS $>14 \mathrm{~d}$ & 9.78 & 8.57 & 14.68 \\
\hline Pneumonia in hospital & 4.29 & 3.76 & 6.46 \\
\hline Septicemia in hospital & 1.70 & 1.47 & 2.61 \\
\hline Stroke in hospital $>72 \mathrm{~h}$ & 2.11 & 1.98 & 2.64 \\
\hline Renal failure in hospital & 5.45 & 4.69 & 8.52 \\
\hline Reoperation, any reason & 10.88 & 9.77 & 15.35 \\
\hline Operative mortality & 4.56 & 3.48 & 8.92 \\
\hline
\end{tabular}

Data presented as median (25th to 75 th percentiles) or \%. BMI, Body mass index; ICU, intensive care unit; $L O S$, length of stay. 
APPENDIX TABLE E2. Outcomes by additive valve procedure (mitral and tricuspid)

\begin{tabular}{|c|c|c|c|}
\hline \multirow[b]{2}{*}{ Characteristics } & \multirow[b]{2}{*}{ Overall $(n=13,743)$} & \multicolumn{2}{|c|}{ Concomitant valve procedure (mitral and tricuspid) } \\
\hline & & No $(n=12,697)$ & Yes $(n=1046)$ \\
\hline Age (y) & $58(48-69)$ & $58(48-68)$ & $62(52-73)$ \\
\hline Female gender & 28.82 & 27.79 & 41.40 \\
\hline BMI $\left(\mathrm{kg} / \mathrm{m}^{2}\right)$ & $28(25-32)$ & $28(25-32)$ & $27(24-31)$ \\
\hline Chronic lung disease & 16.55 & 16.14 & 21.61 \\
\hline Diabetes mellitus & 13.03 & 12.90 & 14.72 \\
\hline Any previous cardiovascular surgery & 19.82 & 18.88 & 31.26 \\
\hline Congestive heart failure & 25.85 & 23.59 & 53.35 \\
\hline Atrial fibrillation & 11.40 & 9.96 & 28.87 \\
\hline \multicolumn{4}{|l|}{ Procedure status } \\
\hline Elective & 83.08 & 83.96 & 72.37 \\
\hline Urgent & 16.92 & 16.04 & 27.63 \\
\hline \multicolumn{4}{|l|}{ Urgent reason } \\
\hline Anatomy & 29.11 & 31.12 & 14.88 \\
\hline Valve dysfunction & 37.10 & 36.48 & 41.52 \\
\hline Congestive heart failure & 22.66 & 20.62 & 37.02 \\
\hline Operation time $(\mathrm{h})$ & $5.3(4.3-6.7)$ & $5.2(4.2-6.6)$ & $6.5(5.3-8.3)$ \\
\hline Crossclamp time (min) & $139(110-178)$ & $136(108-173)$ & $187(149-234)$ \\
\hline ICU stay (h) & $45.8(24.0-88.2)$ & $45.0(24.0-76.7)$ & $72.0(41.0-153.5)$ \\
\hline LOS $>14 \mathrm{~d}$ & 9.78 & 8.73 & 22.47 \\
\hline Pneumonia in hospital & 4.29 & 4.00 & 7.84 \\
\hline Septicemia in hospital & 1.70 & 1.58 & 3.15 \\
\hline Renal failure in hospital & 5.45 & 4.83 & 13.00 \\
\hline Dialysis in hospital & 2.31 & 2.06 & 5.26 \\
\hline Cardiac arrest in hospital & 3.38 & 3.13 & 6.31 \\
\hline Reoperation, bleeding or tamponade & 5.80 & 5.55 & 8.80 \\
\hline Reoperation, any reason & 10.88 & 10.25 & 18.45 \\
\hline Operative mortality & 4.56 & 3.84 & 13.29 \\
\hline
\end{tabular}

Data presented as median (25th to 75 th percentiles) or \%. BMI, Body mass index; $I C U$, intensive care unit; $L O S$, length of stay. 
APPENDIX TABLE E3. Outcomes stratified by additional CABG

\begin{tabular}{|c|c|c|c|}
\hline \multirow[b]{2}{*}{ Characteristic } & \multirow[b]{2}{*}{ Overall $(n=13,743)$} & \multicolumn{2}{|c|}{ Concomitant CABG } \\
\hline & & No $(n=10,214)$ & Yes $(n=3525)$ \\
\hline Age (y) & $58(48-69)$ & $55(45-66)$ & $66(58-74)$ \\
\hline Female gender & 28.82 & 28.20 & 30.64 \\
\hline $\mathrm{BMI}\left(\mathrm{kg} / \mathrm{m}^{2}\right)$ & $28(25-32)$ & $28(25-32)$ & $28(25-32)$ \\
\hline Hypertension & 66.42 & 61.56 & 80.48 \\
\hline Current or recent smoker & 17.54 & 16.61 & 20.23 \\
\hline Hypercholesterolemia & 48.90 & 42.33 & 67.91 \\
\hline Stroke, history & 6.61 & 5.65 & 9.39 \\
\hline Peripheral vascular disease & 14.17 & 12.30 & 19.60 \\
\hline Diabetes mellitus & 13.04 & 10.46 & 20.48 \\
\hline Any previous cardiovascular surgery & 19.83 & 20.19 & 18.78 \\
\hline Congestive heart failure & 25.85 & 24.20 & 30.61 \\
\hline \multicolumn{4}{|l|}{ Procedure status } \\
\hline Elective & 83.08 & 85.72 & 75.43 \\
\hline Urgent & 16.92 & 14.28 & 24.57 \\
\hline \multicolumn{4}{|l|}{ Urgent reason } \\
\hline Anatomy & 29.12 & 27.48 & 31.87 \\
\hline Valve dysfunction & 37.12 & 41.60 & 29.56 \\
\hline Congestive heart failure & 22.62 & 24.88 & 18.82 \\
\hline Acute myocardial infarction & 3.14 & 0.82 & 7.04 \\
\hline Operation time $(\mathrm{h})$ & $5.3(4.2-6.8)$ & $5.0(4.1-6.4)$ & $6.3(5.1-7.9)$ \\
\hline Crossclamp time (min) & $139(110-178)$ & $133(105-170)$ & $160(127-198)$ \\
\hline ICU stay (h) & $45.8(24.0-88.2)$ & $43.8(23.9-72.0)$ & $64.5(30.0-121.0)$ \\
\hline $\operatorname{LOS}>14 \mathrm{~d}$ & 9.78 & 7.66 & 15.94 \\
\hline Pneumonia in hospital & 4.29 & 3.36 & 7.01 \\
\hline Septicemia in hospital & 1.70 & 1.18 & 3.18 \\
\hline Renal failure in hospital & 5.45 & 4.04 & 9.53 \\
\hline Dialysis in hospital & 2.31 & 1.60 & 4.37 \\
\hline Cardiac arrest in hospital & 3.38 & 2.43 & 6.13 \\
\hline Reoperation, bleeding or tamponade & 5.80 & 5.06 & 7.94 \\
\hline Reoperation, any reason & 10.88 & 9.09 & 16.09 \\
\hline Operative mortality & 4.55 & 2.86 & 9.45 \\
\hline
\end{tabular}

Data presented as median (25th to 75 th percentiles) or \%. CABG, Coronary artery bypass grafting; $B M I$, body mass index; $I C U$, intensive care unit; $L O S$, length of stay. 
APPENDIX TABLE E4. Outcomes stratified by additional CABG and valve procedure (mitral and tricuspid)

\begin{tabular}{|c|c|c|c|}
\hline \multirow[b]{2}{*}{ Characteristic } & \multirow[b]{2}{*}{ Overall $(n=13,743)$} & \multicolumn{2}{|c|}{ Concomitant CABG and valve procedure (mitral and tricuspid) } \\
\hline & & No $(n=13,444)$ & Yes $(\mathbf{n}=299)$ \\
\hline Age $(y)$ & $58(48-69)$ & $58(48-68)$ & $69(60-75)$ \\
\hline Female gender & 28.82 & 28.48 & 44.15 \\
\hline $\operatorname{BMI}\left(\mathrm{kg} / \mathrm{m}^{2}\right)$ & $28(25-32)$ & $28(25-32)$ & $27(24-32)$ \\
\hline Hypertension & 66.43 & 66.11 & 80.60 \\
\hline Hypercholesterolemia & 48.89 & 48.43 & 69.57 \\
\hline Chronic lung disease & 16.55 & 16.32 & 27.09 \\
\hline Dialysis for renal failure & 0.92 & 0.86 & 3.68 \\
\hline Cerebrovascular disease & 9.18 & 9.01 & 17.06 \\
\hline Diabetes mellitus & 13.03 & 12.79 & 24.08 \\
\hline Any previous cardiovascular surgery & 19.82 & 19.64 & 27.76 \\
\hline Congestive heart failure & 25.85 & 25.17 & 56.52 \\
\hline Atrial fibrillation & 11.40 & 11.04 & 27.76 \\
\hline \multicolumn{4}{|l|}{ Procedure status } \\
\hline Elective & 83.08 & 83.43 & 67.22 \\
\hline Urgent & 16.92 & 16.57 & 32.78 \\
\hline \multicolumn{4}{|l|}{ Urgent reason } \\
\hline Anatomy & 29.11 & 29.67 & 16.33 \\
\hline Valve dysfunction & 37.10 & 37.16 & 35.71 \\
\hline Congestive heart failure & 22.66 & 22.13 & 34.69 \\
\hline Operation time (h) & $5.3(4.3-6.8)$ & $5.3(4.3-6.7)$ & $7.4(6.2-8.8)$ \\
\hline Crossclamp time (min) & $139(110-178)$ & $138(109-176)$ & $204(162-253)$ \\
\hline ICU stay $(\mathrm{h})$ & $45.8(24.0-88.2)$ & $45.4(24.0-84.0)$ & $105.0(46.0-185.0)$ \\
\hline $\operatorname{LOS}>14 \mathrm{~d}$ & 9.78 & 9.32 & 30.43 \\
\hline Pneumonia in hospital & 4.29 & 4.20 & 8.70 \\
\hline Septicemia in hospital & 1.70 & 1.62 & 5.02 \\
\hline Renal failure in hospital & 5.45 & 5.20 & 16.72 \\
\hline Dialysis in hospital & 2.31 & 2.20 & 7.02 \\
\hline Cardiac arrest in hospital & 3.38 & 3.27 & 8.36 \\
\hline Stroke in hospital $>72 \mathrm{~h}$ & 2.11 & 2.07 & 4.01 \\
\hline Reoperation, bleeding or tamponade & 5.80 & 5.71 & 9.70 \\
\hline Reoperation, any reason & 10.88 & 10.61 & 22.74 \\
\hline Operative mortality & 4.56 & 4.18 & 21.40 \\
\hline
\end{tabular}

APPENDIX TABLE E5. Biostented (porcine or pericardial) trends

\begin{tabular}{|c|c|c|c|c|c|c|}
\hline Year & Overall & Mechanical & Biostented & Stentless & Homograft & Valve sparing \\
\hline 2004 & 686 & 37.8 & 7.6 & 35.4 & 9.0 & 10.2 \\
\hline 2005 & 1916 & 37.3 & 7.2 & 38.0 & 4.6 & 12.9 \\
\hline 2006 & 2252 & 33.9 & 8.3 & 38.3 & 4.2 & 15.3 \\
\hline 2007 & 2337 & 37.4 & 9.8 & 33.0 & 2.3 & 17.5 \\
\hline 2008 & 2399 & 39.6 & 13.5 & 28.3 & 3.3 & 15.3 \\
\hline 2009 & 2488 & 37.4 & 13.8 & 30.5 & 3.1 & 15.3 \\
\hline
\end{tabular}

Data presented as \%. 